\title{
On classification of Mori contractions: Elliptic curve case
}

\author{
Yuri G. Prokhorov

\begin{abstract}
We study Mori's three-dimensional contractions $f: X \rightarrow$ $Z$. It is proved that on the "good" model $(\bar{X}, \bar{S})$ there are no elliptic components of Diff $\bar{S}$ with coefficients $\geq 6 / 7$.
\end{abstract}

\section{Introduction}

1.1. Let $f: X \rightarrow Z \ni o$ be an extremal log terminal contraction over $\mathbb{C}$, that is:

$X$ is a normal algebraic $\mathbb{Q}$-factorial threefold with at worst log terminal singularities, $f$ is a projective morphism such that $f_{*} \mathcal{O}_{X}=\mathcal{O}_{Z}$, $\rho(X / Z)=1$ and $-K_{X}$ is $f$-ample.

We assume that $\operatorname{dim}(Z) \geq 1$ and regard $(Z \ni o)$ as a sufficiently small Zariski neighborhood. Such contractions naturally appear in the Minimal Model Program [KMM]. By $\operatorname{Exc}(f) \subset X$ denote the exceptional locus of $f$.

According to the general principle introduced by Shokurov [Sh] all such contractions can be divided into two classes: exceptional and nonexceptional. A contraction $f: X \rightarrow Z \ni o$ such as in 1.1] is said to be exceptional if for any complement $K_{X}+D$ near $f^{-1}(o)$ there is at most one divisor $E$ of the function field $\mathcal{K}(X)$ with discrepancy $a(E, D)=-1$. The following is a particular case of the theorem proved in [Sh1] and [P2] (see also [PSh]).

Theorem 1.2. Notation as above. Assume that $f: X \rightarrow Z \ni o$ is nonexceptional. Then for some $n \in\{1,2,3,4,6\}$ there is a member $F \in\left|-n K_{X}\right|$ such that the pair $\left(X, \frac{1}{n} F\right)$ is log canonical near $f^{-1}(o)$.

This work was partially supported by grants INTAS-OPEN-97-2072 and RFFI 99-01-01132. 
Thus nonexceptional contractions have a "good" member in | $n K_{X} \mid, n \in\{1,2,3,4,6\}$. The most important case is the case of Mori contractions, i. e. when $X$ has only terminal singularities:

Conjecture 1.3. Notation as in 1.1. Assume that $X$ has at worst terminal singularities. Then $f: X \rightarrow Z \ni o$ is nonexceptional.

Similar to the classification of three-dimensional terminal singularities, this fact should be the key point in the classification Mori contractions. For example it is very helpful in the study of three-dimensional

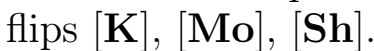

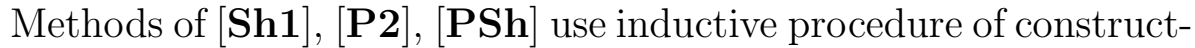
ing divisors in $|-n K|$. This procedure works on so-called good model of $X$ over $Z$. Roughly speaking, a good model is a birational model $\bar{Y}$ equipped with a prime divisor $\bar{S}$ such that the pair $(\bar{Y}, \bar{S})$ is plt and $-\left(K_{\bar{Y}}+\bar{S}\right)$ is nef and big over $Z$.

If $f$ is exceptional, then $\bar{S}$ is a projective surface. Adjunction Formula 2.1 gives us that $(\bar{S}$, Diff $\bar{S})$ is a klt log del Pezzo surface. Moreover, exceptionality of $f$ implies that the projective log pair $\left(\bar{S}, \operatorname{Diff}_{\bar{S}}\right)$ is $e x$ ceptional, by definition this means that any complement $K_{\bar{S}}+\operatorname{Diff}_{\bar{S}}^{+}$is klt [P2, Prop. 2.4]. Thus our construction gives the following correspondence:

$$
\begin{array}{|l}
\text { Exceptional contractions } \\
f: X \rightarrow Z \text { as in } 1.1
\end{array} \rightarrow \begin{aligned}
& \text { Exceptional log del Pezzos } \\
& \left(\bar{S}, \Delta=\operatorname{Diff}_{\bar{S}}\right)
\end{aligned}
$$

1.4. For exceptional log del Pezzos $(\bar{S}, \Delta)$ Shokurov introduced the following invariant:

$$
\begin{aligned}
\delta=\delta(\bar{S}, \Delta)= & \text { number of divisors } E \text { of } \mathcal{K}(\bar{S}) \\
& \text { with discrepancy } a(E, \Delta) \leq-6 / 7
\end{aligned}
$$

He proved that $\delta \leq 2$, classified $\log$ surfaces with $\delta=2$ and showed that in the case $\delta=1$ the (unique) divisor $E$ with $a(E, \Delta) \leq-6 / 7$ is represented by a curve of arithmetical genus $\leq 1$ (see $[$ Sh1 $],[\mathbf{P 3}]$ ).

The aim of this short note is to exclude the case of Mori contractions with $\delta=1$ and elliptic curve $E$ :

TheOREm 1.5. Notation as in 1.1. Assume that $\delta\left(\bar{S}, \operatorname{Diff}_{\bar{S}}\right)=1$. Write Diff $\bar{S}_{\bar{S}}=\sum \delta_{i} \bar{\Delta}_{i}$, where $\bar{\Delta}_{i}$ are irreducible curves. If $\delta_{i_{0}} \geq 6 / 7$ for some $i_{0}$, then $p_{a}\left(\bar{\Delta}_{i_{0}}\right)=0$.

The following example shows that Theorem 1.5 cannot be generalized to the klt case. 
EXAMPLE $1.6(\|\mathbb{I P}\|)$. Let $(Z \ni o)$ be the hypersurface canonical singularity $x_{1}^{2}+x_{3}^{3}+x_{3}^{11}+x_{4}^{12}=0$ and let $f: X \rightarrow Z$ be the weighted blowup with weights $(66,44,12,11)$. Then $f$ satisfies conditions of 1.1 and we have case 3.2. It was computed in [IP] that $S$ is the weighted projective plane $\mathbb{P}(3,2,1)$ and $\operatorname{Diff}_{S}=\frac{10}{11} C+\frac{1}{2} L$, where $C$ is an elliptic curve.

Log del Pezzo surfaces of elliptic type (like $\left(\bar{S}, \operatorname{Diff}_{\bar{S}}\right)$ in the above theorem) were classified by T. Abe $\mathrm{Ab}$. Our proof uses different, very easy arguments.

\section{Preliminary results}

In this paper we use terminologies of Minimal Model Program [KMM], UUt]. For the definition of complements and their proper-

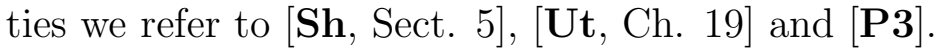

Definition 2.1 ([Sh, Sect. 3], [Ut, Ch. 16]). Let $X$ be a normal variety and let $S \neq \varnothing$ be an effective reduced divisor on $X$. Let $B$ be a $\mathbb{Q}$-divisor on $X$ such that $S$ and $B$ have no common components. Assume that $K_{X}+S$ is lc in codimension two. Then the different of $B$ on $S$ is defined by

$$
K_{S}+\left.\operatorname{Diff}_{S}(B) \equiv\left(K_{X}+S+B\right)\right|_{S} .
$$

Usually we will write simply Diff ${ }_{S}$ instead of $\operatorname{Diff}_{S}(0)$.

TheOREm 2.2 (Inversion of Adjunction [Ut, 17.6]). Let $X$ be a normal variety and let $D$ be a boundary on $X$. Write $D=S+B$, where $S=\lfloor D\rfloor$. Assume that $K_{X}+S+B$ is $\mathbb{Q}$-Cartier. Then $(X, S+B)$ is plt near $S$ iff $S$ is normal and $\left(S, \operatorname{Diff}_{S}(B)\right)$ is klt.

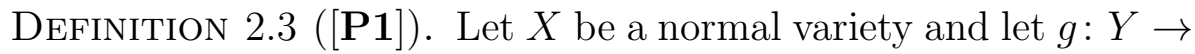
$X$ be a birational contraction such that the exceptional locus of $g$ contains exactly one irreducible divisor, say $S$. Assume that $K_{Y}+S$ is plt and $-\left(K_{Y}+S\right)$ is $f$-ample. Then $g:(Y \supset S) \rightarrow X$ is called a plt blowup of $X$.

The key point in the proof of Theorem 1.5 is the following proposition.

Proposition 2.4. Let $(X \ni P)$ be a three-dimensional terminal singularity and let $g:(Y, S) \rightarrow X$ be a plt blowup with $f(S)=P$. Write $\operatorname{Diff}_{S}=\sum \delta_{i} \Delta_{i}$, where $\Delta_{i}$ are irreducible curves, and assume that $\delta_{0} \geq 6 / 7$ for some $i_{0}$. Further, assume that $S$ is smooth at singular points of $\Delta_{i_{0}}$. Then $p_{a}\left(\Delta_{i_{0}}\right)=0$. 
Lemma 2.5 (cf. [ $[\overline{\mathbf{P 1}}$, Cor. 5]). Let $(X \ni P)$ be a three-dimensional terminal singularity and let $g:(Y, S) \rightarrow X$ be a plt blowup with $f(S)=$ $P$. Then there is a boundary $\Upsilon \geq \operatorname{Diff}_{S}$ on $S$ such that

(i) $\lfloor\Upsilon\rfloor \neq 0$;

(ii) $-\left(K_{S}+\Upsilon\right)$ is ample.

Moreover, $K_{S}+\operatorname{Diff}_{S}$ has a non-klt 1, 2, 3, 4, or 6-complement.

Proof. Regard $(X \ni P)$ as an analytic germ. It was shown in $\left[\mathbf{Y P G}\right.$, Sect. 6.4] that the general element $F \in\left|-K_{X}\right|$ has a normal Du Val singularity at $P$. By Inversion of Adjunction 2.2, $K_{X}+F$ is plt. Consider the crepant pull-back $K_{Y}+a S+F_{Y}=f^{*}\left(K_{X}+F\right)$, where $F_{Y}$ is the proper transform of $F$ and $a<1$. Since both $K_{Y}+S$ and $g^{*} K_{X}$ are $\mathbb{Q}$-Cartier, so are $S$ and $F_{Y}$. Clearly, $-\left(K_{Y}+S+F_{Y}\right)$ is $f$-ample. Let $\Upsilon^{\prime}:=\operatorname{Diff}_{S}\left(F_{Y}\right)$. Then $\lfloor\Upsilon\rfloor^{\prime} \neq 0$ and $-\left(K_{S}+\Upsilon^{\prime}\right)$ is ample. Therefore $\Upsilon$ can be found in the form $\Upsilon=\operatorname{Diff}_{S}\left(F_{Y}\right)+t\left(\Upsilon^{\prime}-\operatorname{Diff}_{S}\left(F_{Y}\right)\right)$ for suitable $0<t \leq 1$.

Take $\Delta=\operatorname{Diff}_{S}\left(F_{Y}\right)+\lambda\left(\Upsilon-\operatorname{Diff}_{S}\left(F_{Y}\right)\right)$ for $0<\lambda \leq 1$ so that $K_{S}+\Delta$ is lc but not klt (and $-\left(K_{S}+\Delta\right)$ is ample). By [Sh1, Sect. 2] (see also $[\overline{\mathbf{P 3}}, 5.4 .1])$ there exists either an 1, 2, 3, 4, or 6-complement of $K_{S}+\Delta$ which is not klt.

A very important problem is to prove the last lemma without using [YPG, Sect. 6.4], i.e. the classification of terminal singularities. This can be a way in higher-dimensional generalizations.

Proof of Proposition 2.4. Put $C:=\Delta_{i_{0}}$ and let $\delta_{0}=1-1 / m$, $m \geq 7$. Assume that $p_{a}(C) \geq 1$. Let $\Upsilon$ be such as in Lemma 2.5 and let $K_{S}+\Theta$ be a non-klt 1, 2, 3, 4, or 6-complement of $K_{S}+$ Diff $_{S}$. Using that the coefficients of Diff $_{S}$ are standard [Sh, Prop. 3.9] it is easy to see that $\Theta \geq \operatorname{Diff}_{S}$ and $\Theta \geq C[\mathbb{\mathbf { P } 3}$, Sect. 4.7]. In particular, $K_{S}+C$ is lc.

Further, $C \not \subset\lfloor\Upsilon\rfloor$. Indeed, otherwise by Adjunction we have

$$
-\operatorname{deg} K_{C} \geq-\operatorname{deg}\left(K_{C}+\operatorname{Diff}_{C}(\Upsilon-C)\right)=-\left(K_{S}+\Upsilon\right) \cdot C>0
$$

This implies $p_{a}(C)=0$, a contradiction.

By Lemma 2.6 below, $\Theta=C, p_{a}(C)=1, S$ is smooth along $C$ and has only Du Val singularities outside. Therefore, Diff ${ }_{S}=(1-1 / m) C$ and $-K_{S} \equiv C \equiv-m\left(K_{S}+(1-1 / m) C\right.$ ) is ample (see 2.3). Thus $S$ is a del Pezzo surface with at worst Du Val singularities. Since $C \not \subset\lfloor\Upsilon\rfloor$, we can write $\Upsilon=\alpha C+L+\Upsilon^{o}$, where $L$ is an irreducible 
curve, $1>\alpha \geq 1-1 / m \geq 6 / 7, C \not \subset \operatorname{Supp}\left(\Upsilon^{o}\right)$ and $\Upsilon^{o} \geq 0$. Further,

$$
\begin{aligned}
0<K_{S} \cdot\left(K_{S}+\Upsilon\right)= & K_{S} \cdot\left(K_{S}+\alpha C+L+\Upsilon^{o}\right) \\
& \leq K_{S} \cdot\left((1-\alpha) K_{S}+L\right) \leq \frac{1}{7} K_{S}^{2}+K_{S} \cdot L .
\end{aligned}
$$

Thus $K_{S}^{2}>-7 K_{S} \cdot L \geq 7$.

Let $S^{\text {min }} \rightarrow S$ be the minimal resolution. By Noether's formula, $K_{S}^{2}+\rho\left(S^{\min }\right)=K_{S_{\text {min }}}^{2}+\rho\left(S^{\min }\right)=10$. Thus, $8 \leq K_{S}^{2} \leq 9$ and $\rho\left(S^{\text {min }}\right) \leq$ 2. In particular, $S$ either is smooth or has exactly one singular point which is of type $A_{1}$. By (11),$-K_{S} \cdot L=1$. Similar to (11) we have

$$
0<-\left(K_{S}+\Upsilon\right) \cdot L \leq-(1-\alpha) K_{S} \cdot L-L^{2} .
$$

Hence $L^{2}<1-\alpha \leq 1 / 7$, so $L^{2} \leq 0$. This means that the curve $L$ generates an extremal ray on $S$ and $\rho(S)=2$. Therefore, $S$ is smooth and $K_{S}^{2}=8$. In this case, $S$ is a rational ruled surface $\left(\mathbb{P}^{1} \times \mathbb{P}^{1}\right.$ or $\left.\mathbb{F}_{1}\right)$. Let $\ell$ be a general fiber of the rulling. Then

$$
0<-\left(K_{S}+\alpha C+L+\Upsilon^{o}\right) \cdot \ell \leq-(1-\alpha) K_{S} \cdot \ell-L \cdot \ell \leq \frac{2}{7}-L \cdot \ell .
$$

Hence $L \cdot \ell=0$, so $L$ is a fiber of $S \rightarrow \mathbb{P}^{1}$ and $-K_{S} \cdot L=2$. This contradicts (11).

Lemma 2.6 (see [P3, Lemma 8.3.6]). Let $(S, C+\Xi)$ be a rational projective log surface, where $C$ is the reduced and $\Xi$ is an arbitrary boundary. Assume that $K_{S}+C+\Xi$ is lc, $S$ is smooth at singular points of $C, K_{S}+C+\Xi \equiv 0, C$ is connected and $p_{a}(C) \geq 1$. Then $\Xi=0$, $K_{S}+C \sim 0, S$ is smooth along $C$ and has only DuVal singularities outside.

Proof. By Adjunction, $K_{C}+\operatorname{Diff}_{C}(\Xi)=0$. Hence Diff $C(\Xi)=0$. This shows that $C \cap \operatorname{Supp}(\Xi)=\varnothing$ and $S$ has no singularities at points of $C \backslash \operatorname{Sing}(C)$ (see [Ut, Prop. 16.6, Cor. 16.7]). Let $\mu: \tilde{S} \rightarrow S$ be the minimal resolution and let $\tilde{C}$ be the proper transform of $C$ on $\tilde{S}$. Define $\tilde{\Xi}$ as the crepant pull-back: $K_{\tilde{S}}+\tilde{C}+\tilde{\Xi}=\mu^{*}\left(K_{S}+C+\Xi\right)$. It is sufficient to show that $\tilde{\Xi}=0$. Assume the converse. Replace $(S, C+\Xi)$ with $(\tilde{S}, \tilde{C}+\tilde{\Xi})$. It is easy to see that all the assumptions of the lemma holds for this new $(S, C+\Xi)$. Contractions of $(-1)$-curves again preserve the assumptions. Since $C$ and $\operatorname{Supp}(\Xi)$ are disjoint, whole $\Xi$ cannot be contracted. Thus we get $S \simeq \mathbb{P}^{2}$ or $S \simeq \mathbb{F}_{n}$ (a rational ruled surface). In both cases simple computations gives us $\Xi=0$. 


\section{Construction of a good model}

Notation as in 1.1. We recall briefly the construction of [P2] (see also [PSh]). Assume that $f: X \rightarrow Z \ni o$ is exceptional. Let $K_{X}+F$ be a complement which is not klt. There is a divisor $S$ of $\mathcal{K}(X)$ such that $a(S, F)=-1$. Since $f$ is exceptional, this divisor is unique.

3.1. First we assume that the center of $S$ on $X$ is a curve or a point. Then $\lfloor F\rfloor=0$. Let $g: Y \rightarrow X$ be a minimal log terminal modification of $(X, F) \llbracket \mathbf{U t}, 17.10]$, i. e. $g$ is a birational projective morphism such that $Y$ is $\mathbb{Q}$-factorial and $K_{Y}+S+A=g^{*}\left(K_{X}+F\right)$ is dlt, where $A$ is the proper transform of $F$. In our situation, $K_{Y}+S+A$ is plt. By [Ut, Prop. 2.17], $K_{Y}+S+(1+\varepsilon) A$ is also plt for sufficiently small positive $\varepsilon$.

3.1.1. Run $(K+S+(1+\varepsilon) A)$-Minimal Model Program over $Z$ :

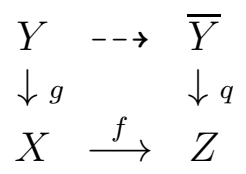

Note that $K_{\bar{Y}}+\bar{S}+(1+\varepsilon) \bar{A} \equiv \varepsilon \bar{A} \equiv-\varepsilon\left(K_{\bar{Y}}+\bar{S}\right)$. At the end we get so-called good model, i. e. a log pair $(\bar{Y}, \bar{S}+\bar{A})$ such that one of the following holds:

(3.1.A) $\rho(\bar{Y} / Z)=2$ and $-\left(K_{\bar{Y}}+\bar{S}\right)$ is nef and big over $Z$;

(3.1.B) $\rho(\bar{Y} / Z)=1$ and $-\left(K_{\bar{Y}}+\bar{S}\right)$ is ample over $Z$.

3.2. Now assume that the center of $S$ on $X$ is of codimension one. Then $S=\lfloor F\rfloor$. In this case, we put $g=\mathrm{id}, Y=X$ and $A=F-S$. If $-\left(K_{X}+S\right)$ is nef, then we also put $\bar{Y}=X, \bar{S}=S$. Assume $-\left(K_{X}+S\right) \equiv A$ is not nef. Since $A$ is effective, $f$ is birational. If $f$ is divisorial, then it must contract a component of $\operatorname{Supp}(A)$. Thus $S$ is not a compact surface, a contradiction (see [ᄑ2, Prop. 2.2]). Therefore $f$ is a flipping contraction. In this case, in diagram (2) the map $X=Y \rightarrow \bar{Y}$ is the corresponding flip.

Since $f$ is exceptional, in both cases 3.1 and 3.2 we have $f(g(S))=$ $q(\bar{S})=o($ see $[\overline{\mathbf{P 2}}$, Prop. 2.2]). Adjunction Formula 2.1 gives us that $\left(\bar{S}\right.$, Diff $\left._{\bar{S}}\right)$ is a klt log del Pezzo surface, i. e. $\left(\bar{S}\right.$, Diff $\left._{\bar{S}}\right)$ is klt and $-\left(K_{\bar{S}}+\right.$ $\operatorname{Diff}_{\bar{S}}$ ) is nef and big (see [P2, Lemma 2.4]). Moreover, exceptionality of $f$ implies that the pair $\left(\bar{S}, \operatorname{Diff}_{\bar{S}}\right)$ is exceptional, i. e. any complement $K_{\bar{S}}+\operatorname{Diff}_{\bar{S}}^{+}$is klt.

Proposition 3.3. Let $f: X \rightarrow Z \ni$ o be as in 1.1. Assume that $f$ is exceptional. Furthermore,

(i) if $f$ is divisorial, we assume that the point $(Z \ni o)$ is terminal; 
(ii) in the case $\operatorname{dim}(Z)=1$, we assume that singularities of $X \backslash$ $f^{-1}(o)$ are canonical.

Then case (3.1. B) does not occur.

Proof. Assume the converse. Then $\rho(\bar{Y} / Z)=1$ and $q: \bar{Y} \rightarrow Z$ is also an exceptional contraction as in 1.1. First, we consider the case when $f$ is divisorial. Then $q$ is a plt blowup of a terminal point $(Z \ni o)$ and $q(\bar{S})=o$ (see $\left[\mathbf{P 2}\right.$, Prop. 2.2]). By Lemma 2.5, $\left(\bar{S}, \operatorname{Diff}_{\bar{S}}\right)$ has a non-klt complement. This contradicts [P2, Prop. 2.4]. Clearly, $f$ cannot be a flipping contraction (because, in this case, the map $Y \rightarrow \bar{Y}$ must be an isomorphism in codimension one). If $\operatorname{dim}(Z)=2$, then $q$ is not equidimensional, a contradiction.

Finally, we consider the case $\operatorname{dim}(Z)=1$ (and $\bar{S}$ is the central fiber of $q$ ). Let $\bar{F}$ be a general fiber of $q$ (a del Pezzo surface with at worst $\mathrm{Du}$ Val singularities). Consider the exact sequence

$$
0 \longrightarrow \mathcal{O}_{\bar{Y}}\left(-K_{\bar{Y}}-\bar{F}\right) \longrightarrow \mathcal{O}_{\bar{Y}}\left(-K_{\bar{Y}}\right) \longrightarrow \mathcal{O}_{\bar{F}}\left(-K_{\bar{F}}\right) \longrightarrow 0
$$

By Kawamata-Viehweg Vanishing [KMM, Th. 1-2-5], $R^{1} q_{*} \mathcal{O}_{\bar{Y}}\left(-K_{\bar{Y}}-\right.$ $\bar{F})=0$. Hence there is the surjection

$$
H^{0}\left(\bar{Y}, \mathcal{O}_{\bar{Y}}\left(-K_{\bar{Y}}\right)\right) \longrightarrow H^{0}\left(\bar{F}, \mathcal{O}_{\bar{F}}\left(-K_{\bar{F}}\right)\right) \longrightarrow 0 .
$$

Here $H^{0}\left(\bar{F}, \mathcal{O}_{\bar{F}}\left(-K_{\bar{F}}\right)\right) \neq 0$ (because $-K_{\bar{F}}$ is Cartier and ample). Therefore, $H^{0}\left(\bar{Y}, \mathcal{O}_{\bar{Y}}\left(-K_{\bar{Y}}\right)\right) \neq 0$. Let $\bar{G} \in\left|-K_{\bar{Y}}\right|$ be any member. Take (positive) $c \in \mathbb{Q}$ so that $K_{\bar{Y}}+\bar{S}+c \bar{G}$ is lc and not plt. Clearly $c \leq 1$, so $-\left(K_{\bar{Y}}+\bar{S}+c \bar{G}\right) \equiv-(1-c) K_{\bar{Y}}$ is $q$-nef. By Base Point Free Theorem [KMM, Th. 3-1-1] there is a complement $K_{\bar{Y}}+\bar{S}+c \bar{G}+L$, where $n L \in\left|-n\left(K_{\bar{Y}}+\bar{S}+c \bar{G}\right)\right|$ for sufficiently big and divisible $n$ and this complement is not plt, a contradiction with exceptionality (see [P2, Prop. 2.4]).

\section{Proof of Theorem 1.5}

In this section we use notation and assumptions of 1.1 and Theorem 1.5 .

If $g=\mathrm{id}$, then $Y=X$ and $\bar{Y}$ have only terminal singularities (see 3.2 and [KMM, 5-1-11]). Then $\operatorname{Diff}_{\bar{S}}=0$, a contradiction. From now on we assume that $g \neq$ id. Denote $\bar{C}:=\bar{\Delta}_{i_{0}}$ and let $\delta_{i_{0}}=1-1 / m$. Since $\delta\left(\bar{S}, \operatorname{Diff}_{\bar{S}}\right)=1$, there are no divisors $E \neq \bar{C}$ of $\mathcal{K}(\bar{S})$ with $a(E$, Diff $\overline{\bar{S}}) \leq$ $-6 / 7$. This gives us that $\bar{S}$ is smooth at $\operatorname{Sing}(\bar{C})$ whenever $\operatorname{Sing}(\bar{C}) \neq \varnothing$ (see [ᄑ3, Lemma 9.1.8]). By our assumptions, $\bar{Y}$ is singular along $\bar{C}$. Moreover, at the general point of $\bar{C}$ we have an analytic isomorphism 
(see [Ut, 16.6]):

$$
(\bar{Y}, \bar{S}, \bar{C}) \simeq\left(\mathbb{C}^{3},\left\{x_{3}=0\right\},\left\{x_{1}-\operatorname{axis}\right\}\right) / \mathbb{Z}_{m}(0,1, q), \quad(m, q)=1 .
$$

Lemma 4.1. Notation as above. Assume that $p_{a}(\bar{C}) \geq 1$. Then the map $\bar{Y} \rightarrow Y$ is an isomorphism at the general point of $\bar{C}$. Moreover, if $\bar{P} \in \bar{C}$ is a singular point, then $\bar{Y} \rightarrow Y$ is an isomorphism at $\bar{P}$. In particular, the proper transform $C$ of $\bar{C}$ is a curve with $p_{a}(C) \geq 1$.

4.2. First we show that Lemma 4.1 implies Theorem 1.5. Assume $p_{a}(\bar{C}) \geq 1$. Clearly, $C \subset S$. By Lemma 4.1, $p_{a}(C) \geq 1$ and

$$
\text { \# } \operatorname{Sing}(C) \geq \# \operatorname{Sing}(\bar{C})
$$

From (3), we have $\operatorname{Diff}_{S}=(1-1 / m) C+$ (other terms).

4.3. Consider the case when $g(S)$ is a point. By Lemma 2.5, as in the proof of Proposition 2.4, one can show that there exists 1, 2, 3, 4, or 6-complement of the form $K_{S}+C+$ (other terms) on $S$. By Adjunction, $p_{a}(C)=1$. Therefore, $p_{a}(\bar{C})=1$ and we have equality in (止). Thus $S$ is smooth at $\operatorname{Sing}(C)$ (whenever $\operatorname{Sing}(C) \neq \varnothing$ ). We have a contradiction by Proposition 2.4.

4.4. Consider the case when $g(S)$ is a curve. Note that $S$ is rational (because $\left(\bar{S}, \operatorname{Diff}_{\bar{S}}\right)$ is a klt log del Pezzo, see e. g. [P3, Sect. $5.5])$ and so is $g(S)$. Consider the restriction $g_{S}: S \rightarrow g(S)$. Since $p_{a}(C) \geq 1, C$ is not a section of $g_{S}$. Let $\ell$ be the general fiber of $g_{S}$. Then $\ell \simeq \mathbb{P}^{1}$ and

$$
2=-K_{S} \cdot \ell>\operatorname{Diff}_{S} \cdot \ell \geq(1-1 / m) C \cdot \ell \geq \frac{6}{7} C \cdot \ell .
$$

Thus $C \cdot \ell=2$ and $C$ is a 2-section of $g_{S}$. Moreover,

$$
\left(\text { Diff }_{S}-(1-1 / m) C\right) \cdot \ell<2-2(1-1 / m)=2 / m<1 / 2 .
$$

Hence Diff $S_{S}$ has no horizontal components other than $C$. Let $P:=$ $g(\ell)$, let $X^{\prime}$ be a germ of a general hyperplane section through $P$ and let $Y^{\prime}:=g^{-1}\left(X^{\prime}\right)$. Consider the induced (birational) contraction $g^{\prime}: Y^{\prime} \rightarrow X^{\prime}$. Since singularities of $X$ are isolated, $X^{\prime}$ is smooth. By Bertini Theorem, $K_{X^{\prime}}+\ell$ is plt. Further, $Y^{\prime}$ has exactly two singular points $C \cap \ell$ and these points are analytically isomorphic to $\mathbb{C}^{2} / \mathbb{Z}_{m}(1, q)$ (see (3)). This contradicts the following lemma.

Lemma 4.5 (see $\mathbb{P 3}$, Sect. 6]). Let $\phi: Y^{\prime} \rightarrow X^{\prime} \ni o^{\prime}$ be a birational contraction of surfaces and let $\ell:=\phi^{-1}\left(o^{\prime}\right)_{\mathrm{red}}$. Assume that $K_{Y^{\prime}}+\ell$ is plt and $X^{\prime} \ni o^{\prime}$ is smooth. Then $Y^{\prime}$ has on $\ell$ at most two singular points. Moreover, if $Y^{\prime}$ has on $\ell$ exactly two singular points, 
then these are of types $\frac{1}{m_{1}}\left(1, q_{1}\right)$ and $\frac{1}{m_{2}}\left(1, q_{2}\right)$, where $\operatorname{gcd}\left(m_{i}, q_{i}\right)=1$ and $\operatorname{gcd}\left(m_{1}, m_{2}\right)=1$.

Proof. We need only the second part of the lemma. So we omit the proof of the first part. Let $\operatorname{Sing}\left(Y^{\prime}\right)=\left\{P_{1}, P_{2}\right\}$. We use topological arguments. Regard $Y^{\prime}$ as a analytic germ along $\ell$. Since $\left(X^{\prime} \ni o^{\prime}\right)$ is smooth, $\pi_{1}\left(Y^{\prime} \backslash \ell\right) \simeq \pi_{1}\left(X^{\prime} \backslash\left\{o^{\prime}\right\}\right) \simeq \pi_{1}\left(X^{\prime}\right)=\{1\}$. On the other hand, for a sufficiently small neighborhood $Y^{\prime} \supset U_{i} \ni P_{i}$ the map $\pi_{1}\left(U_{i} \cap \ell \backslash P_{i}\right) \rightarrow \pi_{1}\left(U_{i} \backslash P_{i}\right)$ is surjective (see [K్ Proof of Th. 9.6]). Using Van Kampen's Theorem as in $\mathbb{M 0}, 0.4 .13 .3]$ one can show that

$$
\pi_{1}\left(Y^{\prime} \backslash\left\{P_{1}, P_{2}\right\}\right) \simeq\left\langle\tau_{1}, \tau_{2}\right\rangle /\left\{\tau_{1}^{m_{1}}=\tau_{2}^{m_{2}}=1, \tau_{1} \tau_{2}=1\right\} .
$$

This group is nontrivial if $\operatorname{gcd}\left(m_{1}, m_{2}\right) \neq 1$, a contradiction.

Proof of Lemma 4.1. The map $Y \rightarrow \bar{Y}$ is a composition of $\log$ flips:

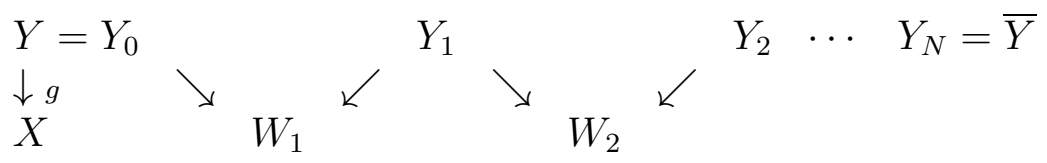

where every contraction $\searrow$ is $(K+S+(1+\varepsilon) A)$-negative and every $\swarrow$ is $(K+S+(1-\varepsilon) A)$-negative. Kawamata-Viehweg Vanishing [KMM, Th. 1-2-5] implies that exceptional loci of these contractions are trees of smooth rational curves [Mo, Cor. 1.3]. Thus Lemma 4.1 is obvious if the curve $\bar{C}$ is nonrational. From now on we assume that $\bar{C}$ is a (singular) rational curve.

LEMMA 4.6. Notation as above. Let $S_{i}$ be the proper transform of $S$ on $Y_{i}$. If $f$ is not a flipping contraction, then $-S_{i}$ is ample over $W_{i}$ for $i=1, \ldots, N$. In particular, all nontrivial fibers of $Y_{i} \rightarrow W_{i}$ are contained in $S_{i}$.

Proof. We claim that $-S_{i}$ is not nef over $Z$ for $i=1, \ldots, N-1$. Indeed, assume $\operatorname{Exc}(f) \neq f^{-1}(o)$. Take $o^{\prime} \in f(\operatorname{Exc}(f)), o^{\prime} \neq o$ and let $\ell \subset g^{-1}\left(f^{-1}\left(o^{\prime}\right)\right)$ be any compact irreducible curve. Clearly, $Y \rightarrow Y_{i}$ is an isomorphism along $\ell$. Let $\ell_{i}$ be the proper transform of $\ell$ on $Y_{i}$. Since $S \cdot \ell=0$, we have $S_{i} \cdot \ell_{i}=0$. The curve $\ell_{i}$ cannot generate an extremal ray (because extremal contractions on $Y_{1}, \ldots, Y_{N-1}$ are flipping). If $-S_{i}$ is nef over $Z$, then taking into account that $\rho\left(Y_{i} / Z\right)=2$ we obtain $S_{i} \equiv 0$, a contradiction. Thus we may assume that $\operatorname{Exc}(f)=f^{-1}(o)$ is a (prime) divisor. Then the exceptional locus of $Y_{i} \rightarrow Z$ is compact. If $-S_{i}$ is nef, this implies $S_{i}=\operatorname{Exc}\left(Y_{i} \rightarrow Z\right)$. Again we have a contradiction because the proper transform of $\operatorname{Exc}(f)$ does not coincide with $S_{i}$ 
We prove our lemma by induction on $i$. It is easy to see that $-S$ is ample over $W_{0}:=X$. The Mori cone $\overline{N E}\left(Y_{i}\right)$ is generated by two extremal rays. Denote them by $R_{i}$ and $Q_{i}$, where $R_{i}$ (resp. $Q_{i}$ ) corresponds to the contraction $Y_{i} \rightarrow W_{i}$ (resp. $\left.Y_{i} \rightarrow W_{i+1}\right)$. Suppose that our assertion holds on $Y_{i-1}$, i. e. $S_{i-1} \cdot R_{i-1}<0$. By our claim above, $S_{i-1} \cdot Q_{i-1}>0$ and after the flip $Y_{i-1} \rightarrow Y_{i}$ we have $S_{i} \cdot R_{i}<0$. This completes the proof of the lemma.

4.7. Let us consider the case when $f$ is not a flipping contraction. Let $C^{(i)}$ be the proper transform of $\bar{C}$ on $Y_{i}$. If $p_{a}\left(C^{(i)}\right) \geq 1$, then $Y_{i} \rightarrow$ $W_{i}$ cannot contract $C^{(i)}$. Thus $C^{(i+1)}$ is well defined. Now we need to show only that on each step of (5) no flipping curves $\operatorname{Exc}\left(Y_{i} \rightarrow W_{i}\right)$ pass through singular points of $C^{(i)}$. (Then $Y_{i} \rightarrow-Y_{i+1}$ is an isomorphism near singular points of $C^{(i)}$ and we are done). By Lemma 4.6 all flipping curves $\operatorname{Exc}\left(Y_{i} \rightarrow W_{i}\right)$ are contained in $S_{i}$. Therefore we can reduce problem in dimension two. The last claim $\operatorname{Exc}\left(Y_{i} \rightarrow W_{i}\right) \cap \operatorname{Sing}\left(C^{(i)}\right)=$ $\varnothing$ easily follows by the lemma below.

LEMmA 4.8. Let $\varphi: S \rightarrow \widehat{S} \ni \hat{o}$ be a birational contraction of surfaces and let $\Delta=\sum \delta_{i} \Delta_{i}$ be a boundary on $S$ such that $K_{S}+\Delta$ is klt and $-\left(K_{S}+\Delta\right)$ is $\varphi$-ample. Put $\Theta:=\sum_{\delta_{i} \geq 6 / 7} \Delta_{i}$. Assume that $\varphi$ does not contract components of $\Theta$. Then $\Theta$ is smooth on $\varphi^{-1}(\hat{o}) \backslash \operatorname{Sing}(S)$.

Proof. Assume the converse and let $P \in \operatorname{Sing}(\Theta) \cap\left(\varphi^{-1}(\hat{o}) \backslash\right.$ $\operatorname{Sing}(S))$. Let $\Gamma$ be a component of $\varphi^{-1}(\hat{o})$ passing through $P$. Then $\Gamma \simeq \mathbb{P}^{1}$. There is an $n$-complement $\Delta^{+}=\sum \delta_{i}^{+} \Delta_{i}$ of $K_{S}+\Delta$ near

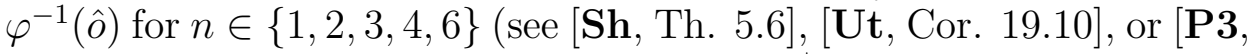
Sect. 6]). By the definition of complements, $\delta_{i}^{+} \geq \min \left\{1,\left\lfloor(n+1) \delta_{i}\right\rfloor / n\right\}$ for all $i$. In particular, $\delta_{i}^{+}=1$ whenever $\delta_{i} \geq 6 / 7$, i. e. $\Theta \leq \Delta^{+}$. This means that $K_{S}+\Theta$ is lc. Since $P \in \operatorname{Sing}(\Theta), K_{S}+\Theta$ is not plt at $P$. Therefore $\Theta=\Delta^{+}$near $P$ and $\Gamma$ is not a component of $\Delta^{+}$. We claim that $K_{S}+\Gamma$ is lc. Indeed, $\Gamma$ is lc at $P$ (because both $\Gamma$ and $S$ are smooth at $P$ ). Assume that $K_{S}+\Gamma$ is not lc at $Q \neq P$. Then $K_{S}+(1-\varepsilon) \Gamma+\Delta^{+}$is not lc at $P$ and $Q$ for $0<\varepsilon \ll 1$. This contradicts Connectedness Lemma [Sh, 5.7], [Ut, 17.4]. Thus $K_{S}+\Gamma$ is lc and we can apply Adjunction:

$$
\left.\left(K_{S}+\Delta^{+}+\Gamma\right)\right|_{\Gamma} \geq\left.\left(K_{S}+\Theta+\Gamma\right)\right|_{\Gamma}=K_{\Gamma}+\operatorname{Diff}_{\Gamma}(\Theta) .
$$

Since $K_{S}+\Delta^{+} \equiv 0$ over $\widehat{S}$ and $\Gamma \simeq \mathbb{P}^{1}$, we have $\operatorname{deg} \operatorname{Diff}_{\Gamma}(\Theta)<2$. On the other hand, the coefficient of $\operatorname{Diff}_{\Gamma}(\Theta)$ at $P$ is equal to $(\Theta \cdot \Gamma)_{P} \geq 2$, a contradiction. 
4.9. Finally let us consider the case when $f$ is a flipping contraction. If $-S_{i}$ is ample over $W_{i}$ for $i=1, \ldots, N$, then we can use arguments of 4.7. From now on we assume that $S_{I}$ is nef over $W_{I}$ for some $1 \leq I \leq N$.

Let $L$ be an effective divisor on $Z$ passing through $o$. Take $c \in \mathbb{Q}$ so that $K_{X}+c f^{*} L$ is lc but not klt. By Base Point Free Theorem [KMM, 3-1-1], there is a member $M \in\left|-n\left(K_{X}+c f^{*} L\right)\right|$ for some $n \in \mathbb{N}$ such that $K_{X}+c f^{*} L+\frac{1}{n} M$ is lc (but not klt). Thus, we may assume $F=c f^{*} L+\frac{1}{n} M$. Let $K_{Y}+S+B^{\prime}=g^{*}\left(K_{X}+c f^{*} L\right)$ be the crepant pull-back. Write $B=B^{\prime}+B^{\prime \prime}$, where $B^{\prime}, B^{\prime \prime} \geq 0$. Then $-\left(K_{Y}+S+B^{\prime}\right)$ is nef over $Z$ and trivial on fibers of $g$. Run $\left(K_{Y}+S+B^{\prime}\right)$-MMP over $Z$. Since $K_{Y}+S+B^{\prime} \equiv-B^{\prime \prime} \neq 0$, this $\mathbb{Q}$-divisor cannot be nef until $S$ is not contracted. Therefore after a number of flips we get a divisorial contraction:

$$
Y \rightarrow Y_{1} \rightarrow \cdots-\rightarrow Y_{N}=\bar{Y} \rightarrow-\rightarrow Y_{N^{\prime}} \longrightarrow X^{\prime} .
$$

Since $\rho\left(Y_{i} / Z\right)=2$ the cone $\overline{N E}\left(Y_{i} / Z\right)$ has exactly two extremal rays. Hence the sequence (5) is contained in (6).

Claim 4.10. $S_{j}$ is nef over $W_{j}$ and $-S_{j}$ is ample over $W_{j+1}$ for $I \leq j \leq N^{\prime}$.

Proof. Clearly, $-S_{I}$ is ample over $W_{I+1}$ (because $S_{I}$ cannot be nef over $Z$ ). After the flip $Y_{I} \rightarrow Y_{I+1}$ we have that $S_{I+1}$ is ample over $W_{I+1}$. Continuing the process we get our claim.

Further, $X^{\prime}$ has only terminal singularities. Indeed, $X^{\prime}$ is $\mathbb{Q}$-factorial, $\rho\left(X^{\prime} / Z\right)=1$ and $X^{\prime} \rightarrow Z$ is an isomorphism in codimension one. Therefore, one of the following holds:

(i) $-K_{X^{\prime}}$ is ample over $Z$, then $X^{\prime} \simeq X$;

(ii) $K_{X^{\prime}}$ is numerically trivial over $Z$, then so is $K_{X}$, a contradiction;

(iii) $K_{X^{\prime}}$ is ample over $Z$, then $X \rightarrow X^{\prime}$ is a flip and $X^{\prime}$ has only terminal singularities [KMM, 5-1-11].

This shows also that $Y_{N^{\prime}} \rightarrow X^{\prime}$ is a plt blowup. Then we can replace $X$ with $X^{\prime}$ and apply arguments of 4.7. This finishes the proof of Theorem 1.5.

Concluding Remark. Shokurov's classification of exceptional log del Pezzos with $\delta \geq 1$ uses reduction to the case $\rho=1$. More precisely, this method uses the following modifications: $\bar{S} \longleftarrow S^{\bullet} \longrightarrow S^{\circ}$, where $S^{\bullet} \rightarrow \bar{S}$ is the blow up of all divisors with discrepancy $a\left(E, \operatorname{Diff}_{\bar{S}} \geq 6 / 7\right.$, $S^{\bullet} \rightarrow S^{\circ}$ is a sequence of some extremal contractions and $\rho\left(S^{\circ}\right)=$ 1. Then all divisors with discrepancy $\geq 6 / 7$ are nonexceptional on $S^{\circ}$. In our case, a smooth elliptic curve with coefficient $\geq 6 / 7$ on $S^{\circ}$ 
cannot be contracted to a point on $\bar{S}$ (because the singularities of $\bar{S}$ are rational). By Theorem 1.5 this case does not occur. The situation in the case of a singular rational curve with coefficient $\geq 6 / 7$ on $S^{\circ}$ which is contracted to a point on $\bar{S}$ is more complicated. This case will be discussed elsewhere.

Acknowledgements. I thank V. V. Shokurov for encouraging me to write up this note. I was working on this problem at Tokyo Institute of Technology during my stay 1999-2000. I am very grateful to the staff of the institute and especially to Professor S. Ishii for hospitality.

\section{References}

[Ab] Abe T. Classification of Exceptional Complements: Elliptic Curve Case, eprint alg-geom/9711029

[HW] Hidaka F., Watanabe K. Normal Gorenstein surfaces with ample anticanonical divisor, Tokyo J. Math. 4 (1981) 319-330

[IP] Ishii S. \& Prokhorov Yu. G. Hypersurface exceptional singularities, preprint TITECH-MATH 06-99 (\#92)

[K] Kawamata, Y., The crepant blowing-up of 3-dimensional canonical singularities and its application to the degeneration of surfaces, Ann. Math. 127 (1988) 93-163

[KMM] Kawamata Y., Matsuda K., Matsuki K. Introduction to the minimal model program, Adv. Stud. Pure Math. 10 (1987) 283-360

[Mo] Mori S. Flip theorem and the existence of minimal models for 3-folds, J. Amer. Math. Soc. 1 (1988) 117-253

[P1] Prokhorov Yu. G. Blow-ups of canonical singularities, Algebra. Proc. Internat. Algebraic Conf. on the Occasion of the 90th Birthday of A. G. Kurosh, Moscow, Russia, May 25-30, 1998, Yu. Bahturin ed., Walter de Gruyter, Berlin (2000), 301-317

[P2] Prokhorov Yu. G. Boundedness of nonbirational extremal contractions, Internat. J. Math (2000) 11 (2000) no. 3, 393-411

[P3] Prokhorov Yu. G. Lectures on complements on log surfaces, e-print math.AG/9912111

[PSh] Prokhorov Yu. G. \& Shokurov V. V. The first main theorem on complements: from global to local, preprint TITECH-MATH 08-99 (\#94)

[YPG] Reid M. Young persons guide to canonical singularities, in "Algebraic Geometry, Bowdoin, 1985" Proc. Symp. Pure Math. 46 (1987) 345-414

[Sh] Shokurov V. V. 3-fold log flips, Izv. AN SSSR, Ser. mat. 56 (1992), 105201 \& 57 (1993), 141-175; English transl. Russian Acad. Sci. Izv. Math. 40 (1993), 93-202 \& 43 (1994), 527-558

[Sh1] Shokurov V. V. Complements on surfaces, e-print, alg-geom/9711024

[Ut] Kollár J. et al. Flips and abundance for algebraic threefolds, A summer seminar at the University of Utah, Salt Lake City, 1991. Astérisque. 211 (1992)

E-mail address: prokhoro@mech.math.msu.su

Department of Mathematics (Algebra Section), Moscow UniverSITY, 117234 Moscow, Russia 